\title{
Hip Preservation Techniques
}

\author{
Raju Vaishya $^{1}$
}

Published online: 23 April 2020

(c) Indian Orthopaedics Association 2020

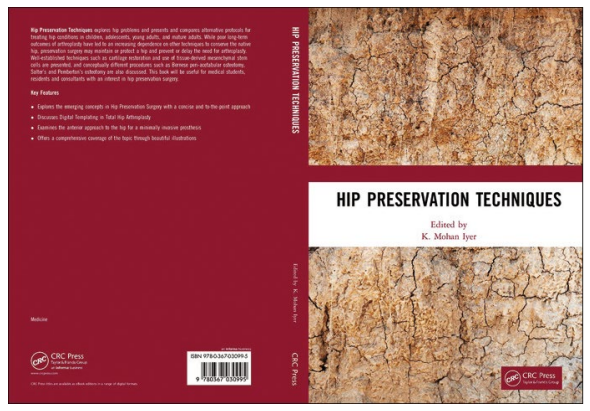

of the hip to provide comfort to the patients (especially the younger ones) and to avoid or alleviate the need for arthroplasty, shortly. The foreword of this book has been written by a known hip preservation surgeon, Dr. Ajay Malviya, of Northumbria Healthcare, UK. This book covers a wide variety of surgical procedures of the hip, to conserve or preserve the hip joint in younger patients. These procedures range from cartilage restoring techniques, mesenchymal cell therapy, hip arthroscopy, minimally invasive, and open procedures (including various types of osteotomy for children and young adults for hip dysplasia, the nonunion of the proximal femur, avascular necrosis, and hip osteoarthritis). The initial part of the book also deals with the background information and the importance of hip preservation and the biomechanics of the hip. This valuable book has specialist contributors in the field of hip preservation from all over the world. I found the book to be unique in its comfortable reading and numerous illustrations and should be quite useful for the medical and postgraduate student, clinicians, and researchers.
Raju Vaishya

raju.vaishya@gmail.com

1 Department of Orthopaedics and Joint Replacement Surgery, Indraprastha Apollo Hospitals, New Delhi, India 